\title{
Prevalência da estenose aterosclerótica do tronco celíaco e da artéria mesentérica superior na arteriopatia oclusiva dos membros inferiores
}

\author{
Prevalence of atherosclerotic stenosis of celiac trunk and superior \\ mesenteric artery in occlusive arteriopathy of lower limbs
}

\author{
Wenes Pereira Reis ${ }^{1}$, Josileide Gaio ${ }^{2}$, César Reis Júnior ${ }^{3}$, Daniel Reis Waisberg ${ }^{4}$, Marly Klug ${ }^{5}$, \\ Robson Barbosa Miranda ${ }^{6}$, Jaques Waisberg ${ }^{7}$
}

\section{Resumo}

Contexto: A eventual relação entre a aterosclerose das artérias dos membros inferiores com a aterosclerose das artérias intestinais foi pouco estudada.

Objetivo: Avaliar pela ecografia vascular (Doppler) presença de lesões com estenose $\geq 70 \%$ na artéria mesentérica superior e/ou tronco celíaco em doentes com arteriopatia obstrutiva crônica dos membros inferiores.

Método: Foram estudados dois grupos, cada um com 60 doentes (40 homens e 20 mulheres). O grupo-caso foi composto por doentes com arteriopatia obstrutiva crônica dos membros inferiores, claudicação intermitente limitante ou dor de repouso e/ou lesões tróficas de extremidade, sem queixas gastrintestinais. O grupo-controle foi constituído por enfermos sem doença arterial obstrutiva dos membros inferiores e sem queixas gastrintestinais. Consideraram-se fatores de risco presença de diabetes melito, hipertensão arterial, obesidade, angina/infarto, tabagismo e dislipidemia. Todos os doentes foram submetidos a ecografia vascular do tronco celíaco e da artéria mesentérica superior. Os doentes do grupo-caso foram separados pela presença de claudicação intermitente limitante $(\mathrm{N}=12)$ ou lesão trófica e/ou dor de repouso $(\mathrm{N}=48)$.

Resultados: Houve associação significante entre idade $(p=0,04) \mathrm{e}$ cardiopatia isquêmica $(p=0,04)$ com aterosclerose da artéria mesentérica superior. Os fatores de risco não mostraram associação significante com presença de estenose do tronco celíaco. Observou-se associação significante entre arteriopatia dos membros inferiores e lesão estenótica da artéria mesentérica superior $(p=0,006)$ e do tronco celíaco $(p<0,001)$.

Conclusões: Na ecografia vascular, o achado de arteriopatia periférica sugere presença de lesão estenótica em artéria mesentérica superior e/ou tronco celíaco. A aterosclerose na artéria mesentérica superior está associada com cardiopatia isquêmica e idade avançada.

Palavras-chave: Estenose, ecografia vascular, artéria mesentérica superior, tronco celíaco.

\begin{abstract}
Background: The occasional relationship between arterial atherosclerosis of lower limbs and atherosclerosis of intestinal arteries has not been fully studied yet.

Objective: To assess the presence of lesions with $\geq 70 \%$ stenosis in the superior mesenteric artery and/or in the celiac trunk in patients with chronic obstructive arteriopathy of the lower limbs using vascular ultrasound (Doppler)

Method: Two groups were involved, a study group and a control group, each comprising 60 patients (40 men and 20 women). The study group consisted of patients with chronic obstructive arterial disease of the lower limbs, limiting intermittent claudication or rest pain, and/or trophic lesions of the extremities but no gastrointestinal complaints. The control group comprised patients without obstructive arterial disease of the lower limbs or gastrointestinal complaints. The presence of diabetes mellitus, arterial hypertension, obesity, angina/infarct, smoking or dyslipidemia was considered a risk factor. All patients were submitted to vascular ultrasound of the celiac trunk and superior mesenteric artery. The patients of the study group were divided according to the presence of limiting intermittent claudication $(n=12)$ or trophic lesion and/or rest pain $(n=48)$.
\end{abstract}

Results: A significant association was found between age $(p=0.04)$ and presence of ischemic cardiopathy $(p=0.04)$ with atherosclerosis of the superior mesenteric artery. The risk factors showed no significant association with presence of stenosis of the celiac trunk. A significant association was found between the presence of arteriopathy of the lower limbs and stenotic lesion of the superior mesenteric artery $(\mathrm{p}=0.006)$ and the celiac trunk $(\mathrm{p}<0.001)$.

Conclusions: On vascular ultrasound, a finding of peripheral arteriopathy of the lower limbs suggests the presence of stenotic lesion in the superior mesenteric artery and /or the celiac trunk. The atherosclerosis in the superior mesenteric artery is associated with ischemic cardiopathy and advanced age.

Keywords: Stenosis, vascular ultrasound, superior mesenteric artery, celiac trunk.

1. Cirurgião Vascular, Serviço de Ultrassonografia, Centro de Terapia e Diagnóstico por Imagem (CIM), Hospital Beatriz Ramos, Indaial SC.

2. Psicóloga, Universidade Regional de Blumenau, Blumenau, SC.

3. Médico residente, Serviço de Cirurgia Geral, Loma Linda University Medical Center, Califórnia, EUA.

4. Acadêmico de Medicina, Faculdade de Medicina da Universidade de São Paulo (FMUSP), São Paulo, SP.

5. Acadêmica de Enfermagem, Universidade Regional de Blumenau, Blumenau, SC.

6. Cirurgião Vascular, Faculdade de Medicina da Fundação ABC, Santo André, SP.

7. Cirurgião, Serviço de Gastroenterologia Cirúrgica, Instituto de Assistência Médica ao Servidor Público Estadual (IAMSPE), São Paulo, SP.

Trabalho realizado no Hospital Beatriz Ramos, Indaial, SC, e no Instituto de Assistência Médica ao Servidor Público Estadual (IAMSPE), São Paulo, SP.

Não foram declarados conflitos de interesse associados à publicação deste artigo.

Artigo submetido em 19.04.09, aceito em 25.11.09.

J Vasc Bras. 2010;9(1):4-13.

Copyright $\odot 2010$ by Sociedade Brasileira de Angiologia e de Cirurgia Vascular 


\section{Introdução}

Embora a presença de aterosclerose das artérias digestivas seja condição frequentemente observada, é habitual o encontro de doentes assintomáticos com estenose acentuada ou mesmo oclusão completa de artérias digestivas. Isso se deve ao desenvolvimento de rede circulatória profusa de suplência das artérias digestivas entre $\mathrm{si}^{1-3}$.

A natureza sistêmica do processo aterosclerótico contribui para o desenvolvimento de doença concomitante em outros territórios, como o cerebral e o cardíaco ${ }^{4}$. Consequentemente, doentes com aterosclerose nos membros inferiores apresentam risco maior de evento de isquemia miocárdica e infarto, além de acidente vascular cerebral, afecções essas que podem ocasionar a morte ${ }^{4,5}$. Ademais, a aterosclerose é responsável por, aproximadamente, 90\% das lesões estenóticas renais ${ }^{6,7}$.

Doentes com isquemia mesentérica crônica apresentam, frequentemente, acometimento por doença aterosclerótica nos membros inferiores e nas artérias coronárias e carótidas ${ }^{8}$. Entretanto, a relação da aterosclerose das artérias intestinais com a aterosclerose dos membros inferiores tem sido pouco estudada.

Durante muito tempo, o estudo das artérias digestivas foi realizado por meio de autópsias ${ }^{9,10}$ e arteriografias ${ }^{5,11}$. Este último exame, entretanto, apresenta restrições por ser invasivo e por não estar isento de complicações ${ }^{12,13}$.

Estudos da circulação intestinal que compararam a arteriografia e a ecografia vascular com Doppler conferiram credibilidade a este último exame para avaliar a circulação arterial dos membros inferiores e, mais recentemente, das artérias intestinais $^{14-16}$. A ecografia vascular com Doppler é considerada método de execução simples, não invasivo e de baixo custo $^{6,12,17}$, permitindo estudar a circulação das artérias intestinais de maneira menos invasiva e avaliar o fluxo no tronco celíaco (TC) e na artéria mesentérica superior (AMS), além da direção e velocidade do fluxo nessas artérias. A realização do exame pode ser dificultada pela obesidade, superposição de alças intestinais ou pela presença de ascite ${ }^{18-20}$.

Em conformidade com a maioria dos autores ${ }^{6,12,13,21}, \mathrm{o}$ critério mais aceito para diagnóstico de estenose de $70 \%$ ou mais da luz das artérias digestivas com o uso da ecografia vascular com Doppler é o adotado por Moneta et al. ${ }^{16}$, que considera o grau de estenose importante quando, no paciente, a velocidade de pico sistólico for $\geq 200 \mathrm{~cm} / \mathrm{s}$ no TC e $\geq 275 \mathrm{~cm} / \mathrm{s}$ na AMS. Moneta et al. ${ }^{16}$ verificaram que a ausência de fluxo ou a velocidade de pico sistólico $\geq 200 \mathrm{~cm} / \mathrm{s}$ no TC e a ausência de fluxo ou a velocidade de pico sistólico $\geq 275 \mathrm{~cm} / \mathrm{s}$ na AMS revelam segmento arterial com estenose significante.

Este estudo teve como objetivo avaliar, por meio da ecografia vascular com Doppler, a prevalência de lesões oclusivas do TC e da AMS em doentes com arteriopatia oclusiva dos membros inferiores.

\section{Método}

O projeto de pesquisa foi aprovado pelo Comitê de Ética em Pesquisa (CEP) do Hospital do Servidor Público Estadual, São Paulo, SP, e pelo Comitê de Ética em Pesquisa do Hospital Beatriz Ramos, Indaial, SC. O estudo foi realizado de acordo com os padrões éticos aceitos pela Declaração de Helsinki da Associação Médica Mundial, adotado em 1864 e emendado em 1996.

Foram estudadas duas séries de doentes designados como grupo-caso e grupo-controle, ambos constituídos de 60 doentes. O grupo-caso foi composto por doentes que apresentavam arteriopatia oclusiva dos membros inferiores. O grupo-controle foi representado por enfermos sem doença intestinal ou arteriopatia dos membros inferiores diagnosticadas e que foram convidados a participar do presente estudo como voluntários.

Os dois grupos foram pareados de acordo com sexo e idade. Foram avaliados, em cada grupo, 40 indivíduos do sexo masculino e 20 do sexo feminino. A média de idade dos doentes do grupo-caso foi de $66 \pm 11$ anos (39 a 93 anos), enquanto que no grupo-controle a média de idade foi de $65 \pm 10$ anos (36 a 91 anos).

Em cada um dos grupos foram avaliados dados clínico-demográficos (idade, sexo, etnia e presença de doenças pré-existentes), fatores de risco para arteriopatia (diabetes melito, hipertensão arterial sistêmica, obesidade, angina/infarto cardíaco, tabagismo e dislipidemia).

Foram considerados obesos os indivíduos que apresentaram índice de massa corporal $>30\left(\text { peso/altura }{ }^{2}\right)^{22}$.

Foram considerados dislipidêmicos os indivíduos com nível sérico de colesterol total $>200 \mathrm{mg} / \mathrm{dL}$, colesterol LDL $>100 \mathrm{mg} / \mathrm{dL}$, colesterol HDL $<40 \mathrm{mg} / \mathrm{dL}$ e nível sérico de triglicerídeos $>150 \mathrm{mg} / \mathrm{dL}^{23}$. Foram considerados 
hipertensos os indivíduos com pressão arterial sistólica ou diastólica $\geq 140$ e $\geq 90 \mathrm{~mm} \mathrm{Hg}$, respectivamente ${ }^{24}$. Foram classificados como diabéticos os indivíduos com glicemia $>126 \mathrm{mg} / \mathrm{dL}$ após 8 horas de jejum ${ }^{25,26}$.

Todos os doentes do grupo-caso eram sintomáticos no tocante à arteriopatia dos membros inferiores, apresentando claudicação intermitente ou, então, lesões tróficas das extremidades, com ou sem dor de repouso, porém assintomáticos em relação às queixas relacionadas às alterações circulatórias das artérias digestivas. O grupo-controle não possuía doença arterial oclusiva dos membros inferiores e os seus membros também eram assintomáticos em relação à doença arterial oclusiva da circulação digestiva.

No exame físico vascular foi aferida a intensidade das pulsações arteriais dos membros inferiores usando-se o critério subjetivo de 0 a 3 cruzes de intensidade, considerando-se, assim: 0 = ausência de pulsação; $+=$ diminuição importante da pulsação; ++ = diminuição moderada; +++ $=$ pulsação normal $^{27}$. Também foi realizada ausculta no trajeto das artérias dos membros inferiores para pesquisar eventual presença de sopros.

Os indivíduos do grupo-caso foram classificados de acordo com a presença no quadro clínico de claudicação intermitente, ou, por outro lado, lesão trófica e/ou dor de repouso. O primeiro foi constituído por $12(20 \%)$ doentes (oito homens e quatro mulheres), e o segundo, por 48 (80\%) doentes (32 homens e 16 mulheres).

Para caracterizar o grau de isquemia do membro estudado, a pressão arterial (PA) foi medida nos membros superiores e inferiores, obtendo-se, assim, o índice tornozelo-braço, no qual o numerador é a PA sistólica dos tornozelos e o denominador, a PA sistólica do membro superior. $\mathrm{O}$ valor de 0,9 foi considerado o valor normal ${ }^{28}$.

Todos doentes - do grupo-caso e do grupo-controle foram submetidos a ecografia da circulação arterial intestinal utilizando-se aparelho de ecografia vascular com Doppler (modelo HDI 5000, ATL $^{\circledR}$, Bothell, WA, EUA) e transdutores convexos de 2 a $5 \mathrm{MHz}$.

Os doentes foram examinados em posição supina após jejum de 12 horas com o transdutor do aparelho de ecografia na posição correspondente à projeção cutânea do TC e da AMS na região epigástrica em insonação longitudinal. A avaliação com Doppler foi realizada no segmento mais representativo do fluxo da artéria em questão. Foram estu- dados o óstio e os primeiros centímetros do TC e da AMS para análise de fluxo. Velocidades $\geq 200 \mathrm{~cm} / \mathrm{s}$ na TC e $\geq 275 \mathrm{~cm} / \mathrm{s}$ na AMS caracterizavam estenose $\geq 70 \%$ nessas $\operatorname{artérias~}^{16}$. Nos indivíduos do grupo-caso e controle que não apresentavam sinal de fluxo sanguíneo arterial na avaliação ecográfica, configurava-se a presença de oclusão do vaso. A média de duração dos exames ecográficos foi de $14 \pm 1,5$ minutos.

\section{Resultados}

\section{Comparação entre os grupos caso e controle}

Verificou-se que o grupo-caso e o grupo-controle se mostraram homogêneos em relação à idade e sexo. Os doentes do grupo-caso apresentaram maior proporção de indivíduos com diabetes melito, hipertensão arterial e tabagismo quando comparados ao grupo-controle $(\mathrm{p}<0,05)$. Não houve diferença significante na proporção de doentes obesos, dislipidêmicos, com presença de angina e acidente vascular cerebral (AVC) prévio entre o grupo-caso e o controle $(\mathrm{p}>0,05)$ (Tabela 1).

Nenhum dos doentes do grupo-controle apresentou lesão da AMS ou lesão do TC. O grupo-caso apresentou maior proporção de doentes com lesão da AMS ou lesão do TC quando comparado ao grupo-controle $(\mathrm{p}=0,006 \mathrm{e}$ $\mathrm{p}<0,001$, respectivamente). Constatou-se que, independentemente do nível de acometimento da circulação periférica do doente, a maioria (70\%) deles não apresentava lesão de nenhuma das duas artérias avaliadas, e, dessa forma, não houve associação significante entre o acometimento periférico e a lesão arterial intestinal $(\mathrm{p}=0,55)$ (Tabela 2).

Entre os 60 doentes com arteriopatia dos membros inferiores (grupo-caso) foi observada lesão da AMS em oito $(13,3 \%)$ [intervalo de confiança de 95\% (IC95\%) 5,9-24,6\%]. Em relação ao TC, observou-se que dos 60 doentes com arteriopatia dos membros inferiores, $14(23,3 \%)$ apresentaram lesão do TC (IC95\% 13,3-36\%) (Tabela 3).

Observou-se que 42 (70\%) dos doentes com arteriopatia dos membros inferiores não apresentaram lesão da AMS ou do TC (Tabela 3). Aproximadamente quatro (7\%) doentes apresentaram lesão em ambas as artérias avaliadas. Apesar de ser observada maior proporção de doentes com lesão do TC (23\%) quando comparada à proporção observada de lesão da AMS (13\%), essa diferença não se mostrou significante $(\mathrm{p}=0,18)$. 
Tabela 1 - Características clínicas dos doentes dos grupos caso e controle

\begin{tabular}{|c|c|c|c|}
\hline & $\begin{array}{c}\text { Caso } \\
(n=60)\end{array}$ & $\begin{array}{l}\text { Controle } \\
(n=60)\end{array}$ & $\mathrm{p}$ \\
\hline Idade* & $67(61-74)$ & $67(60-74)$ & 0,91 \\
\hline \multicolumn{4}{|l|}{ Sexo } \\
\hline Feminino & $20(33,3 \%)$ & $20(33,3 \%)$ & 1,00 \\
\hline Masculino & $40(66,7 \%)$ & $40(66,7 \%)$ & \\
\hline Diabetes & & & $<0,001 \dagger$ \\
\hline Sim & $29(48,3 \%)$ & $4(6,7 \%)$ & \\
\hline Não & $31(51,7 \%)$ & $56(93,3 \%)$ & \\
\hline Hipertensão & & & $0,001 \dagger$ \\
\hline Sim & $40(66,7 \%)$ & $21(35 \%)$ & \\
\hline Não & $20(33,3 \%)$ & $39(65 \%)$ & \\
\hline Obesidade & & & 0,11 \\
\hline Sim & $15(25 \%)$ & $8(13,6 \%)$ & \\
\hline Não & $45(75 \%)$ & $51(86,4 \%)$ & \\
\hline Angina/Infarto & & & 0,16 \\
\hline Sim & $7(11,7 \%)$ & $2(3,3 \%)$ & \\
\hline Não & $53(88,3 \%)$ & $58(96,7 \%)$ & \\
\hline Tabagismo & & & $<0,001 \dagger$ \\
\hline Sim & $31(51,7 \%)$ & - & \\
\hline Não & $29(48,3 \%)$ & $60(100 \%)$ & \\
\hline Dislipidemia & & & 0,21 \\
\hline Sim & $19(31,7 \%)$ & $13(21,7 \%)$ & \\
\hline Não & $41(68,3 \%)$ & $47(78,3 \%)$ & \\
\hline AVC prévio & & & $>0,99$ \\
\hline Sim & $1(1,7 \%)$ & - & \\
\hline Não & $59(98,3 \%)$ & $60(100 \%)$ & \\
\hline $\begin{array}{l}\text { Claudicação } \\
\text { limitante }\end{array}$ & $11(18,3 \%)$ & - & \\
\hline $\begin{array}{l}\text { Lesão trófica e/ou } \\
\text { dor limitante }\end{array}$ & $49(81,7 \%)$ & - & \\
\hline Lesão AMS & & & $0,006 \dagger$ \\
\hline Sim & $8(13,3 \%)$ & - & \\
\hline Não & $52(86,7 \%)$ & $60(100 \%)$ & \\
\hline Lesão TC & & & $<0,001 \dagger$ \\
\hline Sim & $14(23,3 \%)$ & - & \\
\hline Não & $46(76,7 \%)$ & $60(100 \%)$ & \\
\hline
\end{tabular}

$\mathrm{AMS}=$ artéria mesentérica superior; $\mathrm{AVC}=$ acidente vascular cerebral; $\mathrm{TC}=$ tronco celíaco.

* Mediana ( $1^{\circ}$ quartil ao $3^{\circ}$ quartil).

$\uparrow$ Significante.
Tabela 2 - Distribuição dos doentes com arteriopatia dos membros inferiores de acordo com o grau de acometimento vascular periférico

\begin{tabular}{lccc}
\hline & \multicolumn{2}{c}{ Sintoma periférico } \\
\cline { 2 - 3 } $\begin{array}{l}\text { Lesão da } \\
\text { artéria }\end{array}$ & $\begin{array}{c}\text { Claudicação } \\
\mathrm{N}(\%)\end{array}$ & $\begin{array}{c}\text { Lesão trófica e/ou dor } \\
\text { de repouso } \\
\mathrm{N}(\%)\end{array}$ & $\begin{array}{c}\text { Total } \\
\mathrm{N}(\%)\end{array}$ \\
\hline TC & $3(27,3)$ & $7(14,3)$ & $10(16,6)$ \\
AMS & - & $4(8,2)$ & $4(6,7)$ \\
TC e AMS & - & $4(8,2)$ & $4(6,7)$ \\
Ausente & $8(72,7)$ & $34(69,4)$ & $42(70)$ \\
Total & $11(100)$ & $49(100)$ & $60(100)$ \\
\hline
\end{tabular}

AMS $=$ artéria mesentérica superior; $\mathrm{N}=$ Número de doentes; $\mathrm{TC}=$ Tronco celíaco.

Tabela 3 - Distribuição dos 60 doentes com arteriopatia dos membros inferiores de acordo com presença de lesão da artéria mesentérica superior ou de lesão do tronco celíaco

\begin{tabular}{lccc}
\hline & \multicolumn{2}{c}{ Lesão do TC } & \\
\cline { 2 - 3 } Lesão da & Positivo & Negativo & Total \\
AMS & $\mathrm{N}(\%)$ & $\mathrm{N}(\%)$ & $\mathrm{N}(\%)$ \\
\hline Positivo & $4(6,7)$ & $4(6,7)$ & $8(13,4)$ \\
Negativo & $10(16,6)$ & $42(70)$ & $52(86,6)$ \\
Total & $14(23,3)$ & $46(76,7)$ & $60(100)$ \\
\hline
\end{tabular}

AMS $=$ artéria mesentérica superior; $\mathrm{N}=$ número de doentes; $\mathrm{TC}=$ tronco celíaco.

\section{Artéria mesentérica superior}

Sexo, diabetes melito, hipertensão arterial, obesidade, tabagismo, dislipidemia e presença de quadro clínico não se mostraram associados com a presença de lesão na AMS $(\mathrm{p}>0,05)$ (Tabela 4). No tocante à idade, doentes com presença de lesão da AMS apresentaram mediana de idade significantemente superior $(p=0,04)$ do que a apresentada pelos doentes com ausência de lesão da AMS, indicando que, quando a idade do doente com arteriopatia dos membros inferiores aumenta em 1 ano, a possibilidade de lesão da AMS aumenta aproximadamente $11 \%$. Também foi observada associação significante $(p=0,043)$ entre lesão da AMS e angina/infarto, ou seja, a presença de lesão da AMS em doentes com arteriopatia dos membros inferiores que apresentavam angina/infarto foi 7,2 vezes maior do que a de doentes sem angina/infarto. 
Tabela 4 - Distribuição dos fatores de risco para lesão da artéria mesentérica superior

\begin{tabular}{|c|c|c|c|c|}
\hline & \multicolumn{2}{|c|}{ Lesão da AMS } & \multirow[b]{2}{*}{ OR [IC95\%] } & \multirow[b]{2}{*}{$\mathrm{p}$} \\
\hline & Presente $(\mathrm{N}=8)$ & Ausente $(\mathrm{N}=52)$ & & \\
\hline Idade* & $74(67-77)$ & $66(58-72)$ & $1,105[1,003-1,218]$ & 0,04 \\
\hline Sexo & & & & $>0,99$ \\
\hline Feminino & $3(37,5 \%)$ & $17(32,7 \%)$ & $1,235[0,264-5,786]$ & \\
\hline Masculino & $5(62,5 \%)$ & $35(67,3 \%)$ & & \\
\hline Diabetes & & & & 0,14 \\
\hline Sim & $6(75 \%)$ & $23(44,2 \%)$ & $3,783[0,697-20,526]$ & \\
\hline Não & $2(25 \%)$ & $29(55,8 \%)$ & & \\
\hline Hipertensão & & & & 0,71 \\
\hline Sim & $6(75 \%)$ & $34(65,4 \%)$ & $1,588[0,290-8,687]$ & \\
\hline Não & $2(25 \%)$ & $18(34,6 \%)$ & & \\
\hline Obesidade & & & & 1,00 \\
\hline Sim & $2(25 \%)$ & $13(25 \%)$ & $1,000[0,179-5,579]$ & \\
\hline Não & $6(75 \%)$ & $39(75 \%)$ & & \\
\hline Angina/Infarto & & & & $0,04 \uparrow$ \\
\hline Sim & $3(37,5 \%)$ & $4(7,7 \%)$ & $7,200[1,242-41,749]$ & \\
\hline Não & $5(62,5 \%)$ & $48(92,3 \%)$ & & \\
\hline Tabagismo & & & & $>0,99$ \\
\hline Sim & $4(50 \%)$ & $27(51,9 \%)$ & $0,926[0,209-4,104]$ & \\
\hline Não & $4(50 \%)$ & $25(48,1 \%)$ & & \\
\hline Dislipidemia & & & & 0,25 \\
\hline Sim & $4(50 \%)$ & $15(28,8 \%)$ & $2,467[0,545-11,168]$ & \\
\hline Não & $4(50 \%)$ & $37(71,2 \%)$ & & \\
\hline Manifestações & & & & 0,33 \\
\hline Lesão trófica & $8(100 \%)$ & $41(78,8 \%)$ & Não estimável & \\
\hline Claudicação & - & $11(21,2 \%)$ & & \\
\hline
\end{tabular}

AMS = artéria mesentérica superior; IC95\% = intervalo de confiança de 95\%; $\mathrm{N}=$ número de doentes; OR = odds ratio.

* Mediana $\left(1^{\circ}\right.$ quartil ao $3^{\circ}$ quartil).

$\dagger$ Significante.

Para verificar se idade e angina/infarto se mostravam independentemente associadas à lesão da AMS foi realizada análise multivariada por meio do ajuste de modelo multivariado de regressão logística. Nesse modelo foram inseridas, além de idade e angina/infarto, variáveis com $\mathrm{p}<0,20$.

Ao realizar esse ajuste, observou-se que a presença de diabetes melito não se mostrou variável independentemente associada à presença de lesão da AMS $(\mathrm{p}=0,42)$. Ao se fazer o ajuste levando em consideração apenas idade e angina, nenhuma das duas variáveis foram independentes da presença de lesão da AMS ( $\mathrm{p}=0,118$ e 0,119 , respectiva- mente). Verificou-se que tanto a idade como a presença de angina aumentaram a possibilidade de lesão da AMS ( $p=0,04$ para ambas as variáveis).

\section{Tronco celíaco}

Observou-se que nenhuma das variáveis avaliadas apresentou associação significante com a presença de lesão do TC ( $p>0,05)$ (Tabela 5). Constatou-se que as variáveis idade e obesidade apresentaram tendência de associação com a lesão do TC ( $p=0,06$ e $p=0,09$, respectivamente). Apesar de doentes com lesão no tronco celíaco apresentarem mediana de idade maior do que a de doentes 
com ausência dessa lesão, não houve diferença significante $(\mathrm{p}=0,06)$. Houve maior proporção de doentes obesos entre aqueles com lesão do TC (43\%) quando comparada com a proporção observada entre aqueles com ausência de lesão (20\%), porém essa diferença não foi significante.

Devido à significância inferior a $20 \%$ na avaliação dessas variáveis (idade e obesidade) com lesão do TC, optou-se por realizar ajuste multivariado considerando ambas as variáveis no modelo. Esse ajuste mostrou que nenhuma das duas variáveis foi independentemente associada com lesão do TC ( $p=0,17$ para idade e $\mathrm{p}=0,13$ para obesidade).

\section{Discussão}

O processo aterosclerótico é o principal agente etiológico da isquemia mesentérica crônica, assim como do seu acometimento em outros territórios vasculares simultaneamente dependentes de fatores de risco ${ }^{4-6,8,10,21}$. Apesar de a doença aterosclerótica mesentérica ser condição frequente, sua manifestação clínica é rara ${ }^{29}$.

O presente estudo analisou dois grupos distintos: grupo-caso e grupo-controle. No grupo-caso, todos os doentes eram sintomáticos no tocante à circulação arterial dos membros inferiores e apresentavam claudicação intermitente ou lesões tróficas das extremidades e/ou dor de re-

Tabela 5 - Distribuição dos fatores de risco para lesões do tronco celíaco

\begin{tabular}{|c|c|c|c|c|}
\hline & \multicolumn{2}{|c|}{ Lesão do TC } & \multirow[b]{2}{*}{ OR [IC95\%] } & \multirow[b]{2}{*}{$\mathrm{p}$} \\
\hline & Presente $(n=14)$ & Ausente $(n=46)$ & & \\
\hline Idade* & $72(62-77)$ & $66(58-72)$ & $1,055[0,986-1,128]$ & 0,06 \\
\hline Sexo & & & & 0,51 \\
\hline Feminino & $6(42,9 \%)$ & $14(30,4 \%)$ & $1,714[0,501-5,870]$ & \\
\hline Masculino & $8(57,1 \%)$ & $32(69,6 \%)$ & & \\
\hline Diabetes & & & & 0,45 \\
\hline Sim & $8(57,1 \%)$ & $21(45,7 \%)$ & $1,587[0,475-5,307]$ & \\
\hline Não & $6(42,9 \%)$ & $25(54,3 \%)$ & & \\
\hline Hipertensão & & & & 0,34 \\
\hline Sim & $11(78,6 \%)$ & $29(63 \%)$ & $2,149[0,525-8,804]$ & \\
\hline Não & $3(21,4 \%)$ & $17(37 \%)$ & & \\
\hline Obesidade & & & & 0,09 \\
\hline Sim & $6(42,9 \%)$ & $9(19,6 \%)$ & $3,083[0,853-11,145]$ & \\
\hline Não & $8(57,1 \%)$ & $37(80,4 \%)$ & & \\
\hline Angina/Infarto & & & & 0,33 \\
\hline Sim & $3(21,4 \%)$ & $4(8,7 \%)$ & $2,864[0,557-14,726]$ & \\
\hline Não & $11(78,6 \%)$ & $42(91,3 \%)$ & & \\
\hline Tabagismo & & & & 0,64 \\
\hline $\operatorname{Sim}$ & $8(57,1 \%)$ & $23(50 \%)$ & $1,333[0,399-4,454]$ & \\
\hline Não & $6(42,9 \%)$ & $23(50 \%)$ & & \\
\hline Dislipidemia & & & & 0,33 \\
\hline Sim & $6(42,9 \%)$ & $13(28,3 \%)$ & $1,904[0,552-6,565]$ & \\
\hline Não & $8(57,1 \%)$ & $33(71,7 \%)$ & & \\
\hline Quadro clínico & & & & 0,70 \\
\hline Claudicação & $3(21,4 \%)$ & $8(17,4 \%)$ & $1,295[0,293-5,730]$ & \\
\hline Lesão trófica & $11(78,6 \%)$ & $38(82,6 \%)$ & & \\
\hline
\end{tabular}

$\mathrm{TC}=$ tronco celíaco.

* mediana ( $1^{\circ}$ quartil ao $3^{\circ}$ quartil). 
pouso, porém eram assintomáticos em relação à circulação arterial intestinal. No grupo-controle, os doentes eram assintomáticos quanto à circulação arterial dos membros inferiores e à circulação arterial intestinal.

O estudo de ecografia vascular (Doppler) se limitou aos centímetros iniciais das artérias digestivas por se tratar da região mais acometida ${ }^{10}$.

A proposta do uso da ecografia vascular com Doppler para medida do fluxo nas artérias intestinais ocorreu inicialmente em $1982^{30}$ e, de acordo com Perko ${ }^{17}$, o ultrassom Doppler permite o reconhecimento da velocidade e direção do fluxo pela medida do diâmetro luminal e da velocidade sanguínea no interior dos vasos.

Concomitante às alterações vasculares dos membros inferiores dos doentes do grupo-caso, observou-se estenose acentuada $(\geq 70 \%)$ da AMS em oito $(13,6 \%)$ deles com arteriopatia dos membros inferiores e lesão da AMS (IC95\%). Em relação ao TC, 23,7\% dos 60 doentes com arteriopatia dos membros inferiores apresentaram estenose acentuada ( $\geq 70 \%$ ) (IC95\%) do TC. Resultados semelhantes são encontrados na literatura e indicam o TC como local mais frequente de lesão $5,6,9-11,13$. Outros autores, por sua vez, constataram ser a artéria mesentérica inferior ${ }^{31}$ ou a $\mathrm{AMS}^{32}$ o local mais frequente da lesão. Os estudos de Valentine et al. ${ }^{11} \mathrm{e}$ Widman et al. ${ }^{5}$ utilizaram grupos selecionados de doentes com arteriopatia periférica e citaram frequência de acometimento do TC em 25 e 16,1\% e de acometimento da AMS de 6 e 8\%, respectivamente. Estas estenoses, apesar de assintomáticas devido à suplência circulatória, pouco interferem com o funcionamento normal das artérias digestivas ${ }^{5}$, porém apresentam potencial evolutivo para a obstrução completa. No entanto, para que este quadro se instale, há necessidade da obstrução de dois troncos arteriais sequenciais ${ }^{5}$. No presente estudo, apesar da ocorrência de $30 \%$ de estenose significante em uma das artérias intestinais, nenhum desses doentes apresentava sintomas digestivos. De acordo com Waibel ${ }^{33}$, não existe diferença na sintomatologia abdominal de doentes com estenoses de dois ou até mais ramos viscerais quando comparados com aqueles sem estenoses. Jarvinen et al. ${ }^{10}$ encontraram 15\% de casos de acometimento de, no mínimo, duas artérias digestivas que não eram acompanhados de sintomatologia.
$\mathrm{Na}$ atual série de doentes, verificou-se que $70 \%$ dos doentes da amostra não apresentaram lesão da AMS ou do TC. Aproximadamente 7\% dos doentes apresentaram lesão em ambas as artérias avaliadas. Apesar da maior proporção de doentes com lesão do TC (24\%) em relação com a proporção de lesão da AMS (14\%), essa diferença não se mostrou significante $(\mathrm{p}=0,18)$.

$\mathrm{O}$ aumento da frequência de fatores de risco presentes nos doentes com estenose mesentérica foi relatado por Moawad et al. ${ }^{8}$. Estes autores observaram que, dos doentes com isquemia mesentérica crônica, $75 \%$ eram fumantes, $55 \%$ possuíam doença vascular periférica, 43\% apresentavam doença isquêmica coronariana, $37 \%$ eram hipertensos e $10 \%$, dislipidêmicos.

No presente estudo, no grupo-caso, cerca de $80 \%$ dos doentes tinham lesão trófica e/ou dor de repouso, sendo que a diabetes melito estava presente em $48 \%$ dos enfermos, a hipertensão arterial em $67 \%$ e aproximadamente $52 \%$ deles eram tabagistas.

Hansen et al. ${ }^{6}$ afirmaram que a dor abdominal por oclusão aterosclerótica da AMS reflete doença coronária e isquemia miocárdica. Jarvinen et al. ${ }^{10}$, ao analisarem 120 autópsias consecutivas em vítimas de acidentes de trânsito, observaram que a aterosclerose coronária foi significantemente a modalidade mais importante de aterosclerose associada com a aterosclerose mesentérica.Também no atual estudo foi observada associação significante $(p=0,04)$ entre a presença de lesão da AMS e de angina e/ou infarto: a possibilidade de lesão da AMS em doentes com arteriopatia dos membros inferiores que apresentaram angina e/ou infarto é 7,1 vezes maior do que nos doentes que não apresentaram esses eventos. Não foi observada significância com relação à presença de lesões no TC.

A idade também foi associada à presença de aterosclerose mesentérica ${ }^{6,10,11,13}$. Nesta série, a mediana da idade dos doentes com arteriopatia dos membros inferiores foi de 67 anos (39-93 anos). Observou-se que doentes com lesão da AMS apresentaram mediana de idade significantemente maior $(\mathrm{p}=0,04)$ do que a apresentada pelos doentes com ausência de lesão da mesma artéria; ou seja, quando a idade do doente com arteriopatia dos membros inferiores aumenta em 1 ano, a possibilidade de lesão da AMS aumenta em 11\%. Associação significante não foi encontrada nas lesões do TC. Estudos realizados em necropsias mostraram que as lesões das artérias digestivas tendem a 
crescer progressivamente à medida que aumenta a idade do doente ${ }^{10}$. Isso sugere que, com o avançar da idade dos doentes, começa a haver o esgotamento da capacidade orgânica de suprir as necessidades circulatórias arteriais digestivas, progressivamente limitadas pelas estenoses ${ }^{5}$.

Existe discordância na literatura quanto à frequência de estenoses de artérias digestivas em doentes de ambos os sexos, com relatos de maior incidência em homens ${ }^{29}$, em mulheres $^{2}$ e de igualdade em ambos ${ }^{34,35}$. No presente estudo, quanto ao gênero, o sexo masculino foi o mais frequente $(67 \%)$, porém não se mostrou significante.

É aceito que o diabetes melito ${ }^{36}$, o tabagismo ${ }^{37}$, a hipertensão $0^{38}$ e o aumento dos níveis de colesterol representam fatores de risco para a aterosclerose ${ }^{39}$. Entretanto, na atual série, os fatores de risco sexo, diabetes melito, hipertensão arterial, obesidade, tabagismo, dislipidemia, claudicação intermitente e lesão trófica não se mostraram associados com a presença de lesão na AMS. Também se observou que nenhum dos fatores de risco avaliados apresentou associação significante com a presença de lesão no TC. A presença de lesão em ambas as artérias - TC e AMS - ocorreu em frequência pouco expressiva (7\%), fato também observado por Roboottom e Dubbins ${ }^{13}$.

Verificou-se que o grupo-caso apresentou significantemente maior proporção de indivíduos com diabetes melito, hipertensão arterial e tabagismo quando comparado ao grupo-controle. Tal resultado provavelmente é atribuído ao fato de o grupo-caso ser constituído por doentes selecionados pela arteriopatia aterosclerótica obstrutiva dos membros inferiores.

Não houve diferença significante entre o grupo-caso e controle na proporção de doentes obesos, dislipidêmicos, com presença de angina ou com AVC prévio. Nenhum dos doentes do grupo-controle apresentou lesão da AMS ou lesão do TC. Dessa forma, pode-se dizer que o grupo-caso apresentou significantemente maior proporção de doentes com lesão da AMS ou lesão do TC quando comparado ao grupo-controle $(p<0,001)$. A presença de estenose assintomática em artérias intestinais e associada à doença vascular periférica foi também constatada por Valentine et al. ${ }^{11}$. Esses autores citaram a presença de aterosclerose mesentérica em $29 \%$ dos doentes com lesões vasculares ateroscleróticas nos membros inferiores. Reiner et al. ${ }^{32}$ consideram importante a associação entre a aterosclerose mesentérica e a doença vascular periférica.
Na presente série, constatou-se que, em relação ao acometimento vascular periférico - claudicação e lesão trófica e/ou dor de repouso - observado, independentemente dos sintomas apresentados, $70 \%$ dos doentes não apresentaram lesão significante em nenhuma das duas artérias avaliadas (AMS e TC) e, dessa maneira, não houve associação significante entre a presença de sintomatologia arterial periférica e presença de lesão das artérias mesentéricas.

O presente estudo também constatou que a ecografia vascular com Doppler é de execução simples e rápida, não é invasiva e apresenta elevado índice diagnóstico de lesões com estenose $\geq 70 \%$ do lume da AMS ou do TC.

\section{Conclusões}

$\mathrm{O}$ achado de arteriopatia vascular dos membros inferiores sugere a presença de lesão estenótica da AMS e do TC. O encontro de aterosclerose da AMS na ecografia vascular com Doppler está associado com a presença de cardiopatia isquêmica e idade avançada.

\section{Referências}

1. Boudi FB. Atherosclerosis. Emedicine [revista eletrônica]. 2006 Ago [citado 2006 Ago 10]; [aproximadamente 3 p.]. http://www.emedicine.com/med/topic182.htm.

2. Maffei FHA, Lastória S, Yoshida WB, Rollo HA. Doenças vasculares periféricas. $2^{\mathrm{a}}$ ed. Rio de Janeiro: Medsi; 1995. p. 597-616.

3. Cognet F, Salem DB, Dranssart M, et al. Chronic mesenteric ischemia: imaging and percutaneous treatment. Radiographics. 2002;22:863-80.

4. Hirsch AT, Haskal ZJ, Hertzer NR, et al. ACC/AHA 2005 practice guidelines for the management of patients with peripheral arterial disease (lower extremity, renal, mesenteric, and abdominal aortic): a collaborative report from the American Association for Vascular Surgery/Society for Vascular Surgery, Society for Cardiovascular Angiography and Interventions, Society for Vascular Medicine and Biology, Society of Interventional Radiology, and the ACC/AHA Task Force on Practice Guidelines (Writing Committee to Develop Guidelines for the Management of Patients With Peripheral Arterial Disease): endorsed by the American Association of Cardiovascular and Pulmonary Rehabilitation; National Heart, Lung, and Blood Institute; Society for Vascular Nursing; TransAtlantic Inter-Society Consensus; and Vascular Disease Foundation. Circulation. 2006;113:463-5.

5. Widman A, Speranzini MB,Oliveira IRS, Saad WA. Estenose aterosclerótica dos principais troncos arteriais da aorta abdominal (Prevalência em doentes com arteriopatia oclusiva dos membros inferiores). Arq Gastroenterol. 1998;35:18-25. 
6. Hansen KJ, Wilson DB, Craven TE, et al. Mesenteric artery disease in the elderly. J Vasc Surg. 2004;40:45-52.

7. Safian RD, Textor SC. Renal-artery stenosis. N Engl J Med. 2001;344:431-42.

8. Moawad J, Gewertz BL. Chronic mesenteric ischemia. Surg Clin North Am. 1997;77:357-69.

9. Derrik Jr, Pollard HS, Moore RM. The pattern of atherosclerotic narrowing of the celiac and superior mesenteric arteries. Ann Surg. 1959;149:684-9.

10. Jarvinen O, Laurikka J, Sisto T, et al. Atherosclerosis of the visceral arteries. Vasa. 1995;24:9-14.

11. Valentine RJ, Martin JD, Myers SI, Rossi MB, Clagett P. Asymptomatic celiac and superior mesenteric artery stenosis are more prevalent among patients with unsuspected renal artery stenosis. J Vasc Surg. 1991;14:195-9.

12. Lim Hyo K, Lee Won J, Kim Seung H, et al. Splanchnic arterial stenosis or occlusion: diagnosis at Doppler US. Radiology. 1999;211:405-10.

13. Roobottom CA, Dubbins PA. Significant disease of the celiac and superior mesenteric arteries in asymptomatic patients: predictive value of Doppler sonography. Am J Roentenol. 1993;161:985-8.

14. Zeuchner J, Geitung JT, Lukes P, Göthlin JH. Angiography and colour flow duplex ultrasonography in the evaluation of peripheral ischaemic occlusive arterial disease. Acta Radiol. 1994;35:270-4.

15. Nicholls SC, Kohler TR, Martin RL, Strandness Jr. Use of hemodynamic parameters in the diagnosis of mesenteric insufficiency. J Vasc Surg. 1986;3:507-10.

16. Moneta GL, Yeager RA, Dalman R, Antonovic R, Hall LD, Porter JM. Duplex ultrasound criteria for diagnosis of splanchnic artery stenosis or occlusion. J Vasc Surg. 1991;14:511-20.

17. Perko MJ. Dupplex ultrasound for assessment of superior mesenteric artery blood flow. Eur J Vasc Endovasc Surg. 2001;21:106-17.

18. Jäger KA, Fortner GS, Thiele BL, Strandness DE. Noninvasive diagnosis of intestinal angina. J Clin Ultrassound. 1984;12:588-91.

19. Moneta GL, Lee RW, Yeager RA, Taylor LM, Porter JM. Mesenteric duplex scanning: a blinded prospective study. J Vasc Surg. 1993;17:79-86.

20. Armstrong PA. Visceral duplex scanning: evaluation before and after artery intervention for chronic mesenteric ischemia. Perspect Vasc Surg Endovasc Ther. 2007;19:386-92.

21. Mitchell EL, Moneta GL. Mesenteric duplex scanning. Perspect Vasc Surg Endovasc Ther. 2006;18:175-83.

22. Clinical guidelines on the identification, evaluation and treatment of overweight and obesity in adults. National Institute of Health [revista eletrônica]. 1998 Set; Publication Number 98-4083. [aproximadamente $94 \quad$ p.]. http://www.nhlbi.nih.gov/guidelines/obesity/ob_gdlns.pdf.

23. Diretrizes brasileiras sobre dislipidemias e diretrizes de prevenção da aterosclerose do departamento de aterosclerose da Sociedade Brasileira de Cardiologia. Arq Bras Cardiol. 2001;77(Suppl III):1-4.

24. The sixth report of the Joint National Committee on prevention, detection, evaluation, and treatment of high blood pres- sure. National Institute of Health [revista eletrônica]. 1997 Nov; Publication No. 98-4080: [aproximadamente 84 p.]. http://www.sld.cu/galerias/pdf/servicios/hta/6to._reporte_d el_jnc_usa.pdf.

25. Alberti KG, Zimmet PZ. Definition, diagnosis and classification of diabetes mellitus and its complications. Part 1: diagnosis and classification of diabetes, provisional report of a who consultation. Diabet Med. 1998;15:539-53.

26. Sacks DB, Bruns DE, Goldstein DE, Maclaren NK, McDonald JM, Parrott M. Guidelines and recommendations for laboratory analysis in the diagnosis and management of diabetes mellitus. Clin Chem. 2002;48:436-72.

27. Brito CJ, Sales SA, Loureiro E. Avaliação clínica do paciente com arteriopatia. In: Brito CJ, Duque A, Merlo I, Silva RM, et al. Cirurgia vascular. Rio de Janeiro: Revinter; 2002. p. 181-7.

28. Bellen BV, Zorn GW. Laboratório vascular. In: Brito CJ, Duque A, Merlo I, Silva RM, et al. Cirurgia vascular. Rio de Janeiro: Revinter; 2002. p. 188-206.

29. Marston A, Croft R, Menon GP. Does “intestinal angina” exist? A critical study of obstructed visceral arteries. Br J Surg. 1981;68:316-8.

30. Qamar MI, Read AE, Skidmore R, Evans JM, Wells PNT. Transcutaneous Doppler ultrasound measurement of superior mesenteric artery blood flow in man. Gut. 1986;27:1005.

31. Demos NJ, Bahuth JJ, Urnes PD. Comparative study of arteriosclerosis in the inferior and superior mesenteric arteries. With a case report of gangrene of colon. Ann Surg. 1962;155:599-605.

32. Reiner L, Jimenez FA, Rodrigues SL. Arterosclerosis in the mesenteric circulation: observations and correlations with aortic and coronary arterosclerosis. Am Heart J. 1963;66:200-9.

33. Waibel P. Die häufigkeit von stenosen und verschlüssen der A. coeliaca, mesenterica superior und inferior und ihre klinische bedeutung. Vasa. 1977;6:328-33.

34. Fernandes-Cruz L, Astudillo E, Pera C. Las lesiones ateromatosas de las arterias digestivas: estudio de su frecuencia y de su repercusión sobre el calibre de los vasos. Rev Esp Enferm Apar Dig.1972;28:903-20.

35. Rheudasil JM, Stewart MT, Schellack JV, Smith RB, Salam AA, Perdue GD. Surgical treatment of chronic mesenteric arterial insufficiency. J Vasc Surg. 1988;8:495-500.

36. Maffei FHA, Lastoria F. Ateroclerose obliterante periférica: epidemiologia, fisiopatologia, quadro clínico e diagnóstico. Doenças vasculares periféricas. $3^{\mathrm{a}}$ ed. Rio de Janeiro: Medsi; 2002. p. 1007-24.

37. Krupski WC. The peripheral vascular consequences of smoking. Ann Vasc Surg. 1991;5:291-304.

38. Eyler WR, Clark MD, Garman JE, Rian RL, Meininger DE. Angiography of the renal areas including a comparative study of renal stenosis in patients with and without hypertension. Radiology. 1962;78:879-92.

39. Weitz JI, Byrne J, Clagett GP, et al. Diagnosis and treatment of chronic arterial insufficiency of the lower extremities: a critical review. Circulation. 1996;94:3026-49. 
Correspondência:

Wenes Pereira Reis

Rua das Nações, 77, Nações

CEP 89130-000 - Indaial, SC

Tel.: (47) 3394-2952

Fax: (47) 3382-2587

E-mail: wenesreis@yahoo.com.br

\section{Contribuições dos autores}

Concepção e desenho do estudo: WPR, JW, DRW

Análise e interpretação dos dados: WPR, RM, JW
Coleta de dados: CR Jr., MK

Redação do artigo: JG, WPR

Revisão crítica do texto: WPR, JW, DRW, JG, CR Jr., RM, MK, AS

Aprovação final do artigo*: JW

Análise estatística: AS

Responsabilidade geral pelo estudo: WPR

Informações sobre financiamento: N/A

* Todos os autores leram e aprovaram a versão final submetida ao J Vasc Bras. 